\title{
Andrews-Beck type congruences for overpartitions
}

\author{
Eunmi Kim* \\ Institute of Mathematical Sciences \\ Ewha Womans University \\ Seoul, Republic of Korea \\ eunmi.kim67@gmail.com
}

Submitted: May 26, 2021; Accepted: Feb 11, 2022; Published: Feb 25, 2022

(C) The author. Released under the CC BY-ND license (International 4.0).

\begin{abstract}
We prove Andrews-Beck type congruences for overpartitions concerning the $D$ rank and $M_{2}$-rank. To prove congruences, we establish the generating function for weighted $D$-rank (respectively, $M_{2}$-rank) moment of overpartitions and find a connection with the second $D$-rank (respectively, $M_{2}$-rank) moment for overpartitions.
\end{abstract}

Mathematics Subject Classifications: 11P81, 05A17

\section{Introduction}

Ramanujan's congruences for the partition function $p(n)$ are one of remarkable results in the theory of partitions:

$$
\begin{aligned}
p(5 n+4) \equiv 0 & (\bmod 5), \\
p(7 n+5) \equiv 0 & (\bmod 7), \\
p(11 n+6) \equiv 0 & (\bmod 11),
\end{aligned}
$$

Dyson [8] defined the rank of a partition, which is defined as the largest part minus the number of parts, conjectured combinatorial explanations for the Ramanujan congruences modulo 5 and 7 , and conjectured the existence of a crank function for partitions that could provide a combinatorial proof of Ramanujan's congruences modulo 11. Atkin and Swinnerton-Dyer [3] proved Dyson's conjecture on the rank. Andrews and Garvan [2] found the crank function and proved that the crank explains all Ramanujan congruences modulo 5, 7 and 11 .

* Supported by the Basic Science Research Program through the National Research Foundation of Korea funded by the Ministry of Education (NRF-2020R1I1A1A01065877, NRF-2019R1A6A1A11051177). 
Let $N T(b, k, n)$ be the total number of parts in the partitions of $n$ with rank congruent to $b$ modulo $k$. Beck conjectured surprising congruences for the certain linear combinations among $N T(b, k, b)$. Andrews [1] has confirmed Beck's conjectures: for all non-negative integers $n$,

$$
\begin{aligned}
& N T(1,5,5 n+i)+2 N T(2,5,5 n+i) \\
- & 2 N T(3,5,5 n+i)-N T(4,5,5 n+i) \equiv 0 \quad(\bmod 5)
\end{aligned}
$$

for $i=1$ or 4 , and

$$
\begin{aligned}
& N T(1,7,7 n+i)+2 N T(2,7,7 n+i)+3 N T(3,7,7 n+i) \\
& -3 N T(4,7 n+i)-2 N T(5,7,7 n+i)-N T(6,7,7 n+i) \equiv 0 \quad(\bmod 7)
\end{aligned}
$$

for $i=1$ or $i=5$. A crank version of Beck's conjecture is confirmed by Chern [6]. For example, if $M_{\omega}(b, k, n)$ counts the total number of ones in the partitions of $n$ with crank congruent to $b$ modulo $k$, we have, for all integers $n \geqslant 0$,

$$
M_{\omega}(1,5,5 n+4)+2 M_{\omega}(2,5,5 n+4)-2 M_{\omega}(3,5,5 n+4)-M_{\omega}(4,5,5 n+4) \equiv 0 \quad(\bmod 5) .
$$

In the recent article [7], Chern also provided a list of over 70 Andrews-Beck type congruences involving0 $N T(b, k, b)$ and $M_{\omega}(b, k, n)$.

Now, we will consider overpartition analogue to Andrew-Beck type congruences. Recall that an overpartition is a partition in which the first occurrence of a number may be overlined. For example, the 14 overpartitions of 4 are

$$
\begin{gathered}
4, \overline{4}, 3+1, \overline{3}+1,3+\overline{1}, \overline{3}+\overline{1}, 2+2, \overline{2}+2 \\
2+1+1, \overline{2}+1+1,2+\overline{1}+1, \overline{2}+\overline{1}+1,1+1+1+1, \overline{1}+1+1+1 .
\end{gathered}
$$

For an overpartition $\lambda$ of $n$, the $D$-rank of $\lambda$ [11] is defined as Dyson's rank for ordinary partition,

$$
D-\operatorname{rank}(\lambda)=\ell(\lambda)-\#(\lambda)
$$

and the $M_{2}$-rank of $\lambda[12]$ is defined by

$$
M_{2}-\operatorname{rank}(\lambda)=\left\lceil\frac{\ell(\lambda)}{2}\right\rceil-\#(\lambda)+\#\left(\lambda_{o}\right)-\chi(\lambda)
$$

where $\ell(\lambda)$ is the largest part of $\lambda, \#(\lambda)$ is the number of parts in $\lambda, \#\left(\lambda_{o}\right)$ is the number of odd non-overlined parts of $\lambda$, and $\chi(\lambda)=1$ if the largest part of $\lambda$ is odd and non-overlined and $\chi(\lambda)=0$ otherwise.

Let $\overline{N T}(b, k, n)$ denote the total number of parts in the overpartitions of $n$ with $D$ rank congruent to $b$ modulo $k$ and $\overline{N T 2}(b, k, n)$ denote the total number of parts in the overpartitions of $n$ with $M_{2}$-rank congruent to $b$ modulo $k$. Then the following congruences are proved by Chan-Mao-Osburn [5]: for all $n \in \mathbb{N}$,

$\overline{N T 2}(1,5,5 n+2)+2 \overline{N T 2}(2,5,5 n+2)-2 \overline{N T 2}(3,5,5 n+2)-\overline{N T 2}(4,5,5 n+2) \equiv 0 \quad(\bmod 5)$ 
and

$$
\overline{N T}(1,3,3 n+i)-\overline{N T}(2,3,3 n+i) \equiv \overline{N T 2}(1,3,3 n+i)-\overline{N T 2}(2,3,3 n+i) \quad(\bmod 3)
$$

for $i=0$ or 1 .

In this paper, we prove Andrews-Beck type congruence on $\overline{N T}(b, k, n)$ and $\overline{N T 2}(b, k, n)$ modulo 4 and 8 as follows.

Theorem 1. For all integers $n \geqslant 0$,

$$
\overline{N T}(1,4,2 n+1)+2 \overline{N T}(2,4,2 n+1)+3 \overline{N T}(3,4,2 n+1) \equiv 0 \quad(\bmod 4) .
$$

Theorem 2. For all integers $n \geqslant 0$,

$$
\begin{array}{ll}
\overline{N T 2}(1,4,4 n+1)+2 \overline{N T 2}(2,4,4 n+1)+3 \overline{N T 2}(3,4,4 n+1) \equiv 0 & (\bmod 4), \\
\overline{N T 2}(1,4,4 n+2)+2 \overline{N T 2}(2,4,4 n+2)+3 \overline{N T 2}(3,4,4 n+2) \equiv 0 & (\bmod 4),
\end{array}
$$

and

$$
\begin{aligned}
\overline{N T 2}(1,8,4 n+2)+2 \overline{N T 2}(2,8,4 n+2) & +3 \overline{N T 2}(3,8,4 n+2) \\
+4 \overline{N T 2}(4,8,4 n+2)+5 \overline{N T 2}(5,8,4 n+2)+ & 6 \overline{N T 2}(6,8,4 n+2) \\
& +7 \overline{N T 2}(7,8,4 n+2) \equiv 0 \quad(\bmod 8) .
\end{aligned}
$$

Lastly, we also prove a congruence between $\overline{N T}(b, k, n)$ and $\overline{N T 2}(b, k, n)$.

Theorem 3. For all integers $n \geqslant 0$, we have

$$
\sum_{j=1}^{7} j \overline{N T}(j, 8,4 n+1) \equiv \sum_{j=1}^{7} j \overline{N T 2}(j, 8,4 n+1) \quad(\bmod 8) .
$$

The rest of the paper is organized as follows. In Section 2, we establish the generating function for weighted $D$-rank moment of overpartitions and find a relation with the second $D$-rank moment for overpartitions, from which we can prove Theorem 1. Also, we discover more congruences on $\overline{N T}(b, k, n)$. In Section 3, the generating function for weighted $M_{2^{-}}$ rank moment of overpartitions and a proof of Theorem 2 will be presented. Employing generalized Lambert series identities, we prove the congruence between $\overline{N T}(b, k, n)$ and $\overline{N T 2}(b, k, n)$ in Section 4 .

\section{Weighted $D$-rank moments of overpartitions}

Using standard combinatorial arguments in partition theory as [11, Proposition 1.1], we find that

$$
\bar{R}(x, z, q):=\sum_{n \geqslant 0} \sum_{\lambda \in \bar{P}_{n}} x^{\#(\lambda)} z^{D-\operatorname{rank}(\lambda)} q^{n}=\sum_{n \geqslant 0} \frac{(-1)_{n} x^{n} q^{n(n+1) / 2}}{(z q, x q / z)_{n}}
$$


where $\bar{P}_{n}$ is the set of overpartitions of $n$.

Here and throughout the rest of the paper, we use the standard $q$-series notation,

$$
\begin{aligned}
(a)_{n} & =(a ; q)_{n}:=\prod_{k=1}^{n}\left(1-a q^{k-1}\right), \\
\left(a_{1}, \ldots, a_{m}\right)_{n} & =\left(a_{1}, \ldots, a_{m} ; q\right)_{n}:=\left(a_{1}\right)_{n} \cdots\left(a_{m}\right)_{n},
\end{aligned}
$$

and

$$
\left[a_{1}, \ldots, a_{m}\right]_{n}=\left[a_{1}, \ldots, a_{m} ; q\right]_{n}=\left(a_{1}, q / a_{1}, \ldots, a_{m}, q / a_{m}\right)_{n},
$$

for $n \in \mathbb{N}_{0} \cup\{\infty\}$.

We will give two proofs for Theorem 1. For the first proof of Theorem 1, we will establish the generating function for the weighted $D$-rank moment of overpartitions and compare it with the ordinary and symmetrized $D$-rank moments. Here, the ordinary and symmetrized $D$-rank moments are defined by

$$
\begin{aligned}
\bar{N}_{k}(n) & =\sum_{m \in \mathbb{Z}} m^{k} \bar{N}(m, n), \\
\bar{\eta}_{k}(n) & =\sum_{m \in \mathbb{Z}}\left(\begin{array}{c}
m-\left\lfloor\frac{k-1}{2}\right\rfloor \\
k
\end{array}\right) \bar{N}(m, n),
\end{aligned}
$$

where $\bar{N}(m, n)$ denotes the number of overpartitions of $n$ with $D$-rank $m$.

Theorem 4. We have

$$
\sum_{n \geqslant 0} \sum_{\lambda \in \bar{P}_{n}} \#(\lambda) D-\operatorname{rank}(\lambda) q^{n}=-\sum_{n \geqslant 1} \frac{(-1)_{n} q^{n(n+1) / 2}}{(q)_{n}^{2}} \sum_{m=1}^{n} \frac{q^{m}}{\left(1-q^{m}\right)^{2}},
$$

which implies

$$
\sum_{\lambda \in \bar{P}_{n}} \#(\lambda) D-\operatorname{rank}(\lambda)=-\frac{1}{2} \bar{N}_{2}(n)=-\bar{\eta}_{2}(n)
$$

It follows that

$$
\sum_{n \geqslant 1} \frac{(-1)_{n} q^{n(n+1) / 2}}{(q)_{n}^{2}} \sum_{m=1}^{n} \frac{q^{m}}{\left(1-q^{m}\right)^{2}}=2 \frac{(-q)_{\infty}}{(q)_{\infty}} \sum_{n \geqslant 1} \frac{(-1)^{n+1} q^{n(n+1)}}{\left(1-q^{n}\right)^{2}} .
$$

Proof. Applying $[\partial / \partial x]_{x=1}$ to the generating function $\bar{R}(x, z, q)(2.1)$, we have

$$
\begin{aligned}
\sum_{n \geqslant 0} \sum_{\lambda \in \bar{P}_{n}} \#(\lambda) z^{D-\operatorname{rank}(\lambda)} q^{n} & =\frac{\partial}{\partial x}\left[\sum_{n \geqslant 0} \frac{(-1)_{n} x^{n} q^{n(n+1) / 2}}{(z q, x q / z)_{n}}\right]_{x=1} \\
& =\sum_{n \geqslant 0}\left[\frac{(-1)_{n} x^{n} q^{n(n+1) / 2}}{(z q, x q / z)_{n}} \frac{\partial}{\partial x} \log \left(\frac{x^{n}}{(x q / z)_{n}}\right)\right]_{x=1}
\end{aligned}
$$




$$
\begin{aligned}
& =\sum_{n \geqslant 0} \frac{(-1)_{n} q^{n(n+1) / 2}}{(z q, q / z)_{n}}\left[\frac{n}{x}+\sum_{m=1}^{n} \frac{q^{m}}{z-x q^{m}}\right]_{x=1} \\
& =\sum_{n \geqslant 1} \frac{(-1)_{n} q^{n(n+1) / 2}}{(z q, q / z)_{n}}\left(n+\sum_{m=1}^{n} \frac{q^{m}}{z-q^{m}}\right) .
\end{aligned}
$$

Then if we differentiate it by $z$ and evaluate it at $z=1$, we get

$$
\begin{aligned}
\sum_{n \geqslant 0} \sum_{\lambda \in \bar{P}_{n}} \#(\lambda) D-\operatorname{rank}(\lambda) q^{n} & =\frac{\partial}{\partial z}\left[\sum_{n \geqslant 1} \frac{(-1)_{n} q^{n(n+1) / 2}}{(z q, q / z)_{n}}\left(n+\sum_{m=1}^{n} \frac{q^{m}}{z-q^{m}}\right)\right]_{z=1} \\
& =-\sum_{n \geqslant 1} \frac{(-1)_{n} q^{n(n+1) / 2}}{(q)_{n}^{2}} \sum_{m=1}^{n} \frac{q^{m}}{\left(1-q^{m}\right)^{2}},
\end{aligned}
$$

which proves the first part.

If we apply $\left[\frac{\partial}{\partial z}\left(z \frac{\partial}{\partial z}\right)\right]_{z=1}$ to $\bar{R}(1, z, q)$, then we have the generating function for the second $D$-rank moment as follows.

$$
\begin{aligned}
\sum_{n \geqslant 0} \bar{N}_{2}(n) q^{n} & =\sum_{n \geqslant 0} \sum_{\lambda \in \bar{P}_{n}} D-\operatorname{rank}(\lambda)^{2} q^{n} \\
& =\sum_{n \geqslant 0}\left[\frac{\partial}{\partial z}\left(z \frac{\partial}{\partial z} \frac{(-1)_{n} q^{n(n+1) / 2}}{(z q, q / z)_{n}}\right)\right]_{z=1} \\
& =\sum_{n \geqslant 0}\left[\frac{\partial}{\partial z} \frac{(-1)_{n} z q^{n(n+1) / 2}}{(z q, q / z)_{n}} \sum_{m=1}^{n}\left(\frac{q^{m}}{1-z q^{m}}+\frac{q^{m}}{z q^{m}-z^{2}}\right)\right]_{z=1} \\
& =2 \sum_{n \geqslant 1} \frac{(-1)_{n} q^{n(n+1) / 2}}{(q)_{n}^{2}} \sum_{m=1}^{n} \frac{q^{m}}{\left(1-q^{m}\right)^{2}},
\end{aligned}
$$

by comparing with (2.2), which implies that $\sum_{\lambda \in \bar{P}_{n}} \#(\lambda) D-\operatorname{rank}(\lambda)=-\frac{1}{2} \bar{N}_{2}(n)$. Also, from the following relation between the ordinary and symmetrized $D$-rank moments

$$
\bar{N}_{2 k}(n)=\sum_{j=1}^{k}(2 j) ! S^{*}(k, j) \bar{\eta}_{2 j}(n),
$$

where the sequence $S^{*}(n, k)$ is defined recursively by $S^{*}(n+1, k)=S^{*}(n, k-1)+k^{2} S^{*}(n, k)$ and $S^{*}(1,1)=1, S^{*}(n, k)=0$ for $k \leqslant 0$ or $k>n$, we can see that $\frac{1}{2} \bar{N}_{2}(n)=\bar{\eta}_{2}(n)$. Finally, the generating function of $\bar{\eta}_{2}(n)[10$, Theorem 2.1] gives

$$
\sum_{n \geqslant 1} \frac{(-1)_{n} q^{n(n+1) / 2}}{(q)_{n}^{2}} \sum_{m=1}^{n} \frac{q^{m}}{\left(1-q^{m}\right)^{2}}=2 \frac{(-q)_{\infty}}{(q)_{\infty}} \sum_{n \geqslant 1} \frac{(-1)^{n+1} q^{n(n+1)}}{\left(1-q^{n}\right)^{2}} .
$$

Using the generating function for the weighted $D$-rank moment of overpartitions in Theorem 4, we can give a proof of Theorem 1. 
Proof of Theorem 1. By Theorem 4, we have

$$
\begin{aligned}
& \sum_{n \geqslant 0}(\overline{N T}(1,4, n)+2 \overline{N T}(2,4, n)+3 \overline{N T}(3,4, n)) q^{n} \\
& \equiv-2 \frac{(-q)_{\infty}}{(q)_{\infty}} \sum_{n \geqslant 1} \frac{(-1)^{n+1} q^{n(n+1)}}{\left(1-q^{n}\right)^{2}} \quad(\bmod 4) .
\end{aligned}
$$

Using $(-q)_{\infty} /(q)_{\infty} \equiv 1(\bmod 2)$,

$$
\begin{aligned}
2 \frac{(-q)_{\infty}}{(q)_{\infty}} \sum_{n \geqslant 1} \frac{(-1)^{n} q^{n(n+1)}}{\left(1-q^{n}\right)^{2}} \equiv & 2 \sum_{n \geqslant 1} \frac{(-1)^{n} q^{n(n+1)}}{\left(1-q^{n}\right)^{2}} \quad(\bmod 4) \\
= & 2 \sum_{n \geqslant 1}(-1)^{n} q^{n(n+1)} \sum_{m \geqslant 1} m q^{n(m-1)} \\
= & 2 \sum_{n \geqslant 1} \sum_{m \geqslant 1}(2 m-1) q^{4 n^{2}-2 n+(2 m-2)(2 n-1)}\left(q^{4 n+2 m-2}-1\right) \\
& +2 \sum_{n \geqslant 1} \sum_{m \geqslant 1}(2 m) q^{4 n^{2}-2 n+(2 m-1)(2 n-1)}\left(q^{4 n+2 m-1}-1\right) \\
\equiv & 2 \sum_{n \geqslant 1} \sum_{m \geqslant 1} q^{4 n^{2}-2 n+(2 m-2)(2 n-1)}\left(q^{4 n+2 m-2}-1\right) \quad(\bmod 4),
\end{aligned}
$$

Since the last sum involves only terms where the power of $q$ is even, the result follows.

From Theorem 1, we can have the following congruence for the second $D$-rank moment.

Corollary 5. For all integers $n \geqslant 0$,

$$
\bar{N}_{2}(2 n+1) \equiv 0 \quad(\bmod 2) .
$$

In fact, we prove more detailed results on congruences of $\overline{N T}(b, k, n)$, which also deduce the congruence in Theorem 1.

Theorem 6. For all non-negative integers n,

$$
\begin{aligned}
& \overline{N T}(0,4,4 n+i) \equiv 0 \quad(\bmod 4) \quad \text { for } i=0,2,3 \text {, } \\
& \overline{N T}(2,4, n) \equiv 0 \quad(\bmod 4), \\
& \overline{N T}(2,4,4 n+i) \equiv 0 \quad(\bmod 8) \quad \text { for } i=1,2,3 \text {, } \\
& \overline{N T}(1,4,2 n+1) \equiv 0 \quad(\bmod 4) \text {, } \\
& \overline{N T}(3,4,2 n+1) \equiv 0 \quad(\bmod 4) \text {. }
\end{aligned}
$$

Proof. Applying Proposition 2.2 in [5] (a generalization [1, Theorem 3]) with setting $d=1$ and $e \rightarrow 0$, we can rewrite $(2.1)$ as follows.

$$
\bar{R}(x, z, q)=\sum_{n \geqslant 0} \frac{(-1)_{n} x^{n} q^{n(n+1) / 2}}{(z q, x q / z)_{n}}
$$




$$
=1-\frac{(-x q)_{\infty}}{(x q)_{\infty}} \sum_{n \geqslant 1} \frac{(x q,-1)_{n}}{(-x q)_{n}(q)_{n-1}}(-x)^{n} q^{n(n+1)}\left(\frac{1}{q^{n}\left(1-z q^{n}\right)}+\frac{x / z}{1-x q^{n} / z}\right) .
$$

By expanding the terms involving $z$ in a geometric series as in the proof of [1, Corollary4], we have the generating function of $\overline{N T}(b, k, n)$, for $0 \leqslant b \leqslant k$,

$$
\begin{aligned}
\sum_{n \geqslant 0} & \overline{N T}(b, k, n) q^{n} \\
= & -\frac{\partial}{\partial x}\left[\sum_{n \geqslant 1} \frac{\left(-x q^{n+1}\right)_{\infty}(-1)_{n}}{\left(x q^{n+1}\right)_{\infty}(q)_{n-1}}(-x)^{n} q^{n(n+1)}\left(\frac{q^{(b-1) n}}{1-q^{k n}}+\frac{x^{k-b} q^{(k-1-b) n}}{1-x^{k} q^{k n}}\right)\right]_{x=1} \\
= & -\left[\sum_{n \geqslant 1} \frac{\left(-x q^{n+1}\right)_{\infty}(-1)_{n}}{\left(x q^{n+1}\right)_{\infty}(q)_{n-1}}(-x)^{n} q^{n(n+1)} \frac{q^{n(n+1)+(b-1) n}}{1-q^{k n}} \frac{\partial}{\partial x} \log \left(\frac{\left(-x q^{n+1}\right)_{\infty}}{\left(x q^{n+1}\right)_{\infty}} x^{n}\right)\right]_{x=1} \\
- & {\left[\sum_{n \geqslant 1} \frac{\left(-x q^{n+1}\right)_{\infty}(-1)_{n}}{\left(x q^{n+1}\right)_{\infty}(q)_{n-1}}(-x)^{n} q^{n(n+1)} \frac{x^{k-b} q^{(k-1-b) n}}{1-x^{k} q^{k n}} \frac{\partial}{\partial x} \log \left(\frac{\left(-x q^{n+1}\right)_{\infty}}{\left(x q^{n+1}\right)_{\infty}} \frac{x^{n+k-b}}{1-x^{k} q^{k n}}\right)\right]_{x=1} } \\
= & -2 \frac{(-q)_{\infty}}{(q)_{\infty}} \sum_{n \geqslant 1}(-1)^{n} q^{n(n+1)} \frac{1-q^{n}}{1+q^{n}} \frac{q^{(b-1) n}+q^{(k-1-b) n}}{1-q^{k n}}\left(n+2 \sum_{m>n} \frac{q^{m}}{1-q^{2 m}}\right) \\
& -2 \frac{(-q)_{\infty}}{(q)_{\infty}} \sum_{n \geqslant 1}(-1)^{n} q^{n(n+1)} \frac{1-q^{n}}{1+q^{n}} \frac{q^{(k-1-b) n}}{1-q^{k n}}\left(\frac{k}{1-q^{k n}}-b\right) .
\end{aligned}
$$

Setting $k=4$ and using $(1-x) /(1+x) \equiv 1(\bmod 2)$ yield

$$
\begin{aligned}
\sum_{n \geqslant 0} \overline{N T}(b, 4, n) q^{n} \equiv & 2 \sum_{n \geqslant 1} q^{2 n(2 n-1)} \frac{q^{(b-1)(2 n-1)}+q^{(3-b)(2 n-1)}}{1-q^{4(2 n-1)}} \\
& +2 b \sum_{n \geqslant 1}(-1)^{n} q^{n(n+1)} \frac{q^{(3-b) n}}{1-q^{4 n}} \quad(\bmod 4) .
\end{aligned}
$$

For the case $b=0$, we have

$$
\sum_{n \geqslant 0} \overline{N T}(0,4, n) q^{n} \equiv 2 \sum_{n \geqslant 1} q^{(2 n-1)^{2}} \quad(\bmod 4)
$$

which has only terms with the powers of $q$ congruent 1 modulo 4 . Hence, $\overline{N T}(0,4,4 n+i) \equiv$ $0(\bmod 4)$ for $i=0,2,3$ and all integers $n \geqslant 0$. For the case $b=1$ and 3 , we find that

$$
\sum_{n \geqslant 0} \overline{N T}(b, 4, n) q^{n} \equiv 2 \sum_{n \geqslant 1} q^{2 n(2 n-1)} \frac{q^{(b-1)(2 n-1)}}{1-q^{4(2 n-1)}}+2 \sum_{n \geqslant 1} q^{2 n(2 n+1)} \frac{q^{(3-b)(2 n)}}{1-q^{8 n}} \quad(\bmod 4)
$$

has only even powers of $q$, which implies that $\overline{N T}(1,4,2 n+1) \equiv \overline{N T}(3,4,2 n+1) \equiv 0$ $(\bmod 4)$ for all integers $n \geqslant 0$. Similarly, for the case $b=2$, from (2.3),

$$
\sum_{n \geqslant 0} \overline{N T}(2,4, n) q^{n} \equiv 4 \sum_{n \geqslant 1} q^{2 n(2 n-1)} \frac{q^{2 n-1}}{1-q^{4(2 n-1)}}+4 \sum_{n \geqslant 1}(-1)^{n} q^{n(n+1)} \frac{q^{n}}{1-q^{4 n}} \quad(\bmod 8)
$$




$$
\equiv 4 \sum_{n \geqslant 1} q^{2 n(2 n+1)} \frac{q^{2 n}}{1-q^{8 n}} \quad(\bmod 8)
$$

which includes terms only with the powers of $q$ congruent 0 modulo 4 . This proves that $\overline{N T}(2,4, n) \equiv 0(\bmod 4)$ and $\overline{N T}(2,4,4 n+i) \equiv 0(\bmod 8)$ for $i=1,2,3$ for all integers $n$ greater than 0 .

\section{Weighted $M_{2}$-rank moments of overpartitions}

As in Section 2, we find the generating function for the weighted $M_{2}$-rank moments of overpartitions and compare it with the ordinary and symmetrized $M_{2}$-rank moments. We have the ordinary and symmetrized $M_{2}$-rank moments defined by

$$
\begin{aligned}
\overline{N 2}_{k}(n) & =\sum_{m \in \mathbb{Z}} m^{k} \overline{N 2}(m, n), \\
\overline{\eta 2}_{k}(n) & =\sum_{m \in \mathbb{Z}}\left(\begin{array}{c}
m-\left\lfloor\frac{k-1}{2}\right\rfloor \\
k
\end{array}\right) \overline{N 2}(m, n),
\end{aligned}
$$

where $\overline{N 2}(m, n)$ denotes the number of overpartitions of $n$ with $M_{2}$-rank $m$. The generating function [12, Theorem 1.2] is

$$
\overline{R 2}(x, z, q):=\sum_{n \geqslant 0} \sum_{\lambda \in \bar{P}_{n}} x^{\#(\lambda)} z^{M_{2}-\operatorname{rank}(\lambda)} q^{n}=\sum_{n \geqslant 0} \frac{\left(-1,-q ; q^{2}\right)_{n}(x q)^{n}}{\left(z q^{2}, x q^{2} / z ; q^{2}\right)_{n}}
$$

Theorem 7. We have

$$
\sum_{n \geqslant 0} \sum_{\lambda \in \bar{P}_{n}} \#(\lambda) M_{2}-\operatorname{rank}(\lambda) q^{n}=-\sum_{n \geqslant 1} \frac{\left(-1,-q ; q^{2}\right)_{n} q^{n}}{\left(q^{2} ; q^{2}\right)_{n}^{2}} \sum_{m=1}^{n} \frac{q^{2 m}}{\left(1-q^{2 m}\right)^{2}},
$$

which implies

$$
\sum_{\lambda \in \bar{P}_{n}} \#(\lambda) M_{2}-\operatorname{rank}(\lambda)=-\frac{1}{2} \overline{N 2}_{2}(n)=-\overline{\eta 2}_{2}(n)
$$

It follows that

$$
\sum_{n \geqslant 1} \frac{\left(-1,-q ; q^{2}\right)_{n} q^{n}}{\left(q^{2} ; q^{2}\right)_{n}^{2}} \sum_{m=1}^{n} \frac{q^{2 m}}{\left(1-q^{2 m}\right)^{2}}=2 \frac{(-q)_{\infty}}{(q)_{\infty}} \sum_{n \geqslant 1} \frac{(-1)^{n+1} q^{n(n+2)}}{\left(1-q^{2 n}\right)^{2}}
$$

Proof of Theorem $\%$. If we differentiate $\overline{R 2}(x, z, q)$ by $x$ and evaluate it at $x=1$, we get

$$
\sum_{n \geqslant 0} \sum_{\lambda \in \bar{P}_{n}} \#(\lambda) z^{M_{2}-\operatorname{rank}(\lambda)} q^{n}=\frac{\partial}{\partial x}\left[\sum_{n \geqslant 0} \frac{\left(-1,-q ; q^{2}\right)_{n}(x q)^{n}}{\left(z q^{2}, x q^{2} / z ; q^{2}\right)_{n}}\right]_{x=1}
$$




$$
\begin{aligned}
& =\sum_{n \geqslant 0}\left[\frac{\left(-1,-q ; q^{2}\right)_{n}(x q)^{n}}{\left(z q^{2}, x q^{2} / z ; q^{2}\right)_{n}} \frac{\partial}{\partial x} \log \left(\frac{x^{n}}{\left(x q^{2} / z ; q^{2}\right)_{n}}\right)\right]_{x=1} \\
& =\sum_{n \geqslant 1} \frac{\left(-1,-q ; q^{2}\right)_{n} q^{n}}{\left(z q^{2}, q^{2} / z ; q^{2}\right)_{n}}\left(n+\sum_{m=1}^{n} \frac{q^{2 m}}{z-q^{2 m}}\right) .
\end{aligned}
$$

Then applying $[\partial / \partial z]_{z=1}$ gives

$$
\begin{aligned}
\sum_{n \geqslant 0} \sum_{\lambda \in \bar{P}_{n}} \#(\lambda) M_{2}-\operatorname{rank}(\lambda) q^{n} & =\frac{\partial}{\partial z}\left[\sum_{n \geqslant 1} \frac{\left(-1,-q ; q^{2}\right)_{n} q^{n}}{\left(z q^{2}, q^{2} / z ; q^{2}\right)_{n}}\left(n+\sum_{m=1}^{n} \frac{q^{2 m}}{z-q^{2 m}}\right)\right]_{z=1} \\
& =-\sum_{n \geqslant 1} \frac{\left(-1,-q ; q^{2}\right)_{n} q^{n}}{\left(q^{2} ; q^{2}\right)_{n}^{2}} \sum_{m=1}^{n} \frac{q^{2 m}}{\left(1-q^{2 m}\right)^{2}}
\end{aligned}
$$

which is the first part. Next, to compare with the $M_{2}$-rank moments, when we apply $\left[\frac{\partial}{\partial z}\left(z \frac{\partial}{\partial z}\right)\right]_{z=1}$ to $\overline{R 2}(1, z, q)$, we find that

$$
\begin{aligned}
\sum_{n \geqslant 0} \overline{N 2}_{2}(n) q^{n} & =\sum_{n \geqslant 0} \sum_{\lambda \in \bar{P}_{n}} M_{2}-\operatorname{rank}(\lambda)^{2} q^{n} \\
& =2 \sum_{n \geqslant 1} \frac{\left(-1,-q ; q^{2}\right)_{n} q^{n}}{\left(q^{2} ; q^{2}\right)_{n}^{2}} \sum_{m=1}^{n} \frac{q^{2 m}}{\left(1-q^{2 m}\right)^{2}} .
\end{aligned}
$$

Then the second part follows from comparing (3.1) with (3.2) and the following relation between the ordinary and symmetrized $M_{2}$-rank moments

$$
\overline{N 2}_{2 k}(n)=\sum_{j=1}^{k}(2 j) ! S^{*}(k, j) \overline{\eta 2}_{2 j}(n)
$$

Lastly, we have the last identity by considering the generating function for $\overline{\eta 2}_{2}(n)[10$, Theorem 2.1].

From the generating function for weighted $M_{2}$-rank moment of overpartitions, we can prove Theorem 2 .

Proof of Theorem 2. By Theorem 7, we notice that

$$
\sum_{n \geqslant 0}(\overline{N T 2}(1,4, n)+2 \overline{N T 2}(2,4, n)+3 \overline{N T 2}(3,4, n)) q^{n} \equiv-2 \frac{(-q)_{\infty}}{(q)_{\infty}} \sum_{n \geqslant 1} \frac{(-1)^{n+1} q^{n(n+2)}}{\left(1-q^{2 n}\right)^{2}}
$$

$(\bmod 4)$.

By applying $(-q)_{\infty} /(q)_{\infty} \equiv 1(\bmod 2)$,

$2 \frac{(-q)_{\infty}}{(q)_{\infty}} \sum_{n \geqslant 1} \frac{(-1)^{n} q^{n(n+2)}}{\left(1-q^{2 n}\right)^{2}} \equiv 2 \sum_{n \geqslant 1} \frac{(-1)^{n} q^{n(n+2)}}{\left(1-q^{n}\right)^{2}} \quad(\bmod 4)$ 


$$
\begin{aligned}
& =2 \sum_{n \geqslant 1}(-1)^{n} q^{n(n+2)} \sum_{m \geqslant 1} m q^{2 n(m-1)} \\
& \equiv 2 \sum_{n \geqslant 1} \sum_{m \geqslant 1}\left(q^{4 n(n+1)+8 n(m-1)}-q^{4 n^{2}-1+4(2 n-1)(m-1)}\right) \quad(\bmod 4),
\end{aligned}
$$

which contributes the term with powers congruent to 0 or 3 modulo 4 . Then we have

$$
\begin{array}{ll}
\overline{N T 2}(1,4,4 n+1)+2 \overline{N T 2}(2,4,4 n+1)+3 \overline{N T 2}(3,4,4 n+1) \equiv 0 & (\bmod 4) \\
\overline{N T 2}(1,4,4 n+2)+2 \overline{N T 2}(2,4,4 n+2)+3 \overline{N T 2}(3,4,4 n+2) \equiv 0 & (\bmod 4) .
\end{array}
$$

Also, by Theorem 7, we find that

$$
\sum_{n \geqslant 0} \sum_{j=1}^{7} j \overline{N T 2}(j, 8, n) q^{n} \equiv-2 \frac{(-q)_{\infty}}{(q)_{\infty}} \sum_{n \geqslant 1} \frac{(-1)^{n+1} q^{n(n+2)}}{\left(1-q^{2 n}\right)^{2}} \quad(\bmod 8) .
$$

Using

$$
\frac{(-q)_{\infty}}{(q)_{\infty}} \equiv \frac{(q)_{\infty}}{(-q)_{\infty}} \quad(\bmod 4)=\sum_{k=-\infty}^{\infty}(-1)^{k} q^{k^{2}}
$$

we have

$$
\begin{aligned}
& 2 \frac{(-q)_{\infty}}{(q)_{\infty}} \sum_{n \geqslant 1} \frac{(-1)^{n} q^{n(n+2)}}{\left(1-q^{2 n}\right)^{2}} \\
& \equiv 2\left(1+2 \sum_{k \geqslant 1}(-1)^{k} q^{k^{2}}\right) \sum_{n \geqslant 1} \frac{(-1)^{n} q^{n(n+2)}}{\left(1-q^{2 n}\right)^{2}} \quad(\bmod 8) \\
& =2\left(1+2 \sum_{k \geqslant 1}(-1)^{k} q^{k^{2}}\right) \sum_{n \geqslant 1}(-1)^{n} q^{n(n+2)} \sum_{m \geqslant 1} m q^{2 n(m-1)} \\
& \equiv 2\left(1+2 \sum_{k \geqslant 1}(-1)^{k} q^{k^{2}}\right) \sum_{n \geqslant 1} \sum_{m \geqslant 1}(2 m-1)\left(q^{4 n(n+1)+8 n(m-1)}-q^{4 n^{2}-1+4(2 n-1)(m-1)}\right) \\
& \quad+2 \sum_{n \geqslant 1} \sum_{m \geqslant 1}(2 m)\left(q^{4 n(n+1)+4 n(2 m-1)}-q^{4 n^{2}-1+2(2 n-1)(2 m-1)}\right)
\end{aligned}
$$

Here,

$$
1+2 \sum_{k \geqslant 1}(-1)^{k} q^{k^{2}}
$$

only contributes to terms with powers of $q$ congruent to 0 or 1 modulo 4 , while the factor

$$
\sum_{n \geqslant 1} \sum_{m \geqslant 1}(2 m-1)\left(q^{4 n(n+1)+8 n(m-1)}-q^{4 n^{2}-1+4(2 n-1)(m-1)}\right)
$$

involves terms with powers of $q$ congruent to 0 or 3 modulo 4 . Therefore, the first term in (3.3) dose not have terms with powers of $q$ congruent to 2 modulo 4 . The second term in (3.3) has only terms with powers of $q$ congruent to 0 or 1 modulo 4 . Hence, we obtain

$$
\overline{N T 2}(1,8,4 n+2)+2 \overline{N T 2}(2,8,4 n+2)+3 \overline{N T 2}(3,8,4 n+2)
$$




$$
\begin{aligned}
& +4 \overline{N T 2}(4,8,4 n+2)+5 \overline{N T 2}(5,8,4 n+2) \\
& +6 \overline{N T 2}(6,8,4 n+2)+7 \overline{N T 2}(7,8,4 n+2) \equiv 0 \quad(\bmod 8) .
\end{aligned}
$$

From Theorem 2 and (1.1), we can have congruences for the second $M_{2}$-rank moments as follows.

Corollary 8. For all integers $n \geqslant 0$,

$$
\begin{array}{ll}
\overline{N 2}_{2}(4 n+1) \equiv 0 & (\bmod 2), \\
\overline{N 2}_{2}(4 n+2) \equiv 0 & (\bmod 4), \\
\overline{N 2}_{2}(5 n+2) \equiv 0 & (\bmod 5) .
\end{array}
$$

\section{Proof of Theorem 3}

Let

$$
\varphi(q):=\sum_{n=-\infty}^{\infty} q^{n^{2}} \text { and } \psi(q):=\sum_{n \geqslant 0} q^{n(n+1) / 2} .
$$

Then, by Jacobi's triple product identity and simple $q$-series manipulations, we have

$$
\varphi(q)=\frac{\left(q^{2} ; q^{2}\right)_{\infty}^{5}}{(q)_{\infty}^{2}\left(q^{4} ; q^{4}\right)_{\infty}^{2}}, \quad \varphi(-q)=\frac{(q)_{\infty}^{2}}{\left(q^{2} ; q^{2}\right)_{\infty}}, \quad \text { and } \quad \psi(q)=\frac{\left(q^{2} ; q^{2}\right)_{\infty}^{2}}{(q)_{\infty}}
$$

and

$$
\varphi^{2}(q)=\varphi^{2}\left(q^{2}\right)+4 q \psi^{2}\left(q^{4}\right), \quad \varphi(q) \varphi(-q)=\varphi^{2}\left(-q^{2}\right), \quad \text { and } \quad \psi^{2}(q)=\varphi(q) \psi\left(q^{2}\right) .
$$

We will also use the following identities [4, Theorem 6.1, and 7.1].

$$
\begin{aligned}
\varphi^{4}(-q) & =1+8 \sum_{n \geqslant 1} \frac{n q^{n(n+2)}+q^{n(n+1)}-n q^{n}}{\left(1+q^{n}\right)^{2}}, \\
\psi^{4}(q) & =\sum_{n \geqslant 0} \frac{\left(2 n+1+2 q^{2 n+1}-(2 n+1) q^{4 n+2}\right) q^{2 n^{2}+2 n}}{\left(1-q^{2 n+1}\right)^{2}} .
\end{aligned}
$$

We prove Theorem 3 using generalized Lambert series identities with above theta function identities.

Proof of Theorem 3. Let

$$
a(n)=\sum_{j=1}^{7} j \overline{N T}(j, 8, n)-\sum_{j=1}^{7} j \overline{N T 2}(j, 8, n) .
$$

By Theorem 4 and 7 , we have

$$
\sum_{n \geqslant 0} a(n) q^{n} \equiv-2 \frac{(-q)_{\infty}}{(q)_{\infty}} \sum_{n \geqslant 1} \frac{(-1)^{n+1} q^{n(n+1)}}{\left(1-q^{n}\right)^{2}}+2 \frac{(-q)_{\infty}}{(q)_{\infty}} \sum_{n \geqslant 1} \frac{(-1)^{n+1} q^{n(n+2)}}{\left(1-q^{2 n}\right)^{2}} \quad(\bmod 8)
$$




$$
=\frac{(-q)_{\infty}}{(q)_{\infty}} \sum_{\substack{n=-\infty \\ n \neq 0}}^{\infty}\left[\frac{(-1)^{n} q^{n(n+1)}}{\left(1-q^{n}\right)^{2}}-\frac{(-1)^{n} q^{n(n+2)}}{\left(1-q^{2 n}\right)^{2}}\right] .
$$

We consider the 4-dissection for $\frac{(-q)_{\infty}}{(q)_{\infty}}[9]$,

$$
\begin{aligned}
\frac{(-q)_{\infty}}{(q)_{\infty}}= & \frac{\left(q^{8} ; q^{8}\right)_{\infty}^{19}}{\left(q^{4} ; q^{4}\right)_{\infty}^{14}\left(q^{16} ; q^{16}\right)_{\infty}^{6}}+2 q \frac{\left(q^{8} ; q^{8}\right)_{\infty}^{13}}{\left(q^{4} ; q^{4}\right)_{\infty}^{12}\left(q^{16} ; q^{16}\right)_{\infty}^{2}} \\
& +4 q^{2} \frac{\left(q^{8} ; q^{8}\right)_{\infty}^{7}\left(q^{16} ; q^{16}\right)_{\infty}^{2}}{\left(q^{4} ; q^{4}\right)_{\infty}^{10}}+8 q^{3} \frac{\left(q^{8} ; q^{8}\right)_{\infty}\left(q^{16} ; q^{16}\right)_{\infty}^{6}}{\left(q^{4} ; q^{4}\right)_{\infty}^{8}} .
\end{aligned}
$$

For the summation part in (4.4),

$$
\begin{aligned}
& \sum_{\substack{n=-\infty \\
n \neq 0}}^{\infty}\left[\frac{(-1)^{n} q^{n(n+1)}}{\left(1-q^{n}\right)^{2}}-\frac{(-1)^{n} q^{n(n+2)}}{\left(1-q^{2 n}\right)^{2}}\right] \\
& =2 \sum_{\substack{n=-\infty \\
n \neq 0}}^{\infty} \frac{(-1)^{n} q^{n(n+1)}\left(1+q^{n}+3 q^{2 n}+q^{3 n}\right)}{\left(1-q^{4 n}\right)^{2}} \\
& \equiv 2 \sum_{\substack{n=-\infty \\
n \neq 0}}^{\infty} \frac{(-1)^{n} q^{n(n+1)}\left(1+q^{n}+3 q^{2 n}+q^{3 n}\right)}{\left(1+q^{4 n}\right)^{2}}(\bmod 8)
\end{aligned}
$$

using the fact

$$
\sum_{\substack{n=-\infty \\ n \neq 0}}^{\infty} \frac{(-1)^{n} q^{n(n+1)} q^{m n}}{\left(1-q^{4 n}\right)^{2}}=\sum_{\substack{n=-\infty \\ n \neq 0}}^{\infty} \frac{(-1)^{n} q^{n(n+1)} q^{(6-m) n}}{\left(1-q^{4 n}\right)^{2}}
$$

By letting

$$
F_{a, b}(q):=\sum_{\substack{n=-\infty \\ n \neq 0 \\ n \equiv b}}^{\infty} \frac{(-1)^{n} q^{n(n+a)}}{\left(1+q^{4 n}\right)^{2}} \text { for } a=1,2,3, \text { and } 4
$$

we can have 4-dissection of (4.6). Invoking (4.5) and the dissection of (4.6) into (4.4) and collecting only terms where the power of $q$ is congruent to 1 modulo 4 yield

$$
\begin{aligned}
& \sum_{n \geqslant 0} a(4 n+1) q^{4 n+1} \\
& \equiv 2 \frac{\left(q^{8} ; q^{8}\right)_{\infty}^{13}}{\left(q^{4} ; q^{4}\right)_{\infty}^{12}\left(q^{16} ; q^{16}\right)_{\infty}^{2}}\left[\begin{array}{l}
\frac{\left(q^{8} ; q^{8}\right)_{\infty}^{6}}{\left(q^{4} ; q^{4}\right)_{\infty}^{2}\left(q^{16} ; q^{16}\right)_{\infty}^{4}}\left(F_{4,1}(q)+F_{4,3}(q)\right) \\
+2 q\left(F_{1,0}(q)+F_{1,3}(q)+F_{2,0}(q)+F_{2,2}(q)\right. \\
\left.\left.+3 F_{3,0}(q)+3 F_{3,1}(q)+F_{4,0}(q)+F_{4,2}(q)\right)\right] \quad(\bmod 8) .
\end{array}\right.
\end{aligned}
$$


Noting that

$$
F_{1,0}(q)+F_{3,0}(q)=\sum_{\substack{n=-\infty \\ n \neq 0}}^{\infty} \frac{q^{16 n^{2}+4 n}}{1+q^{16 n}} \quad \text { and } \quad F_{1,3}(q)+F_{3,1}(q)=-\sum_{n=-\infty}^{\infty} \frac{q^{16 n^{2}+28 n+12}}{1+q^{16 n+12}}
$$

it turns out to be

$$
\begin{aligned}
& \sum_{n \geqslant 0} a(4 n+1) q^{n} \\
& \equiv 2 \frac{\left(q^{2} ; q^{2}\right)_{\infty}^{13}}{(q)_{\infty}^{12}\left(q^{4} ; q^{4}\right)_{\infty}^{2}}\left[-\frac{\left(q^{2} ; q^{2}\right)_{\infty}^{6}}{(q)_{\infty}^{2}\left(q^{4} ; q^{4}\right)_{\infty}^{4}} \sum_{n=-\infty}^{\infty} \frac{q^{n^{2}+3 n+1}}{\left(1+q^{2 n+1}\right)^{2}}+2 \sum_{\substack{n=-\infty \\
n \neq 0}}^{\infty} \frac{q^{4 n^{2}+n}}{1+q^{4 n}}\right. \\
& \left.-2 \sum_{n=-\infty}^{\infty} \frac{q^{4 n^{2}+7 n+3}}{1+q^{4 n+3}}+2 \sum_{\substack{n=-\infty \\
n \neq 0}}^{\infty} \frac{q^{n^{2}+n}}{\left(1+q^{2 n}\right)^{2}}+2 \sum_{\substack{n=-\infty \\
n \neq 0}}^{\infty} \frac{q^{n^{2}+2 n}}{\left(1+q^{2 n}\right)^{2}}\right] \quad(\bmod 8) .
\end{aligned}
$$

Replacing $q$ by $q^{4}$ and setting $b_{1}=-1$ and $b_{2}=-q^{3}(r=0, s=2)$ in [4, Theorem 2.1], we obtain that

$$
\frac{\left(q^{4} ; q^{4}\right)_{\infty}^{2}}{\left[-1,-q^{3} ; q^{4}\right]_{\infty}}=\frac{1}{\left[q^{3} ; q^{4}\right]_{\infty}} \sum_{n=-\infty}^{\infty} \frac{q^{4 n^{2}+n}}{1+q^{4 n}}+\frac{1}{\left[q^{-3} ; q^{4}\right]_{\infty}} \sum_{n=-\infty}^{\infty} \frac{q^{4 n^{2}+7 n}}{1+q^{4 n+3}},
$$

which implies

$$
\sum_{\substack{n=-\infty \\ n \neq 0}}^{\infty} \frac{q^{4 n^{2}+n}}{1+q^{4 n}}-\sum_{n=-\infty}^{\infty} \frac{q^{4 n^{2}+7 n+3}}{1+q^{4 n+3}}=\frac{(q)_{\infty}^{2}\left(q^{4} ; q^{4}\right)_{\infty}^{5}}{2\left(q^{2} ; q^{2}\right)_{\infty}^{3}\left(q^{8} ; q^{8}\right)_{\infty}^{2}}-\frac{1}{2}=\frac{1}{2} \varphi(-q) \varphi\left(q^{2}\right)-\frac{1}{2}
$$

Similarly, replacing $q$ by $q^{2}$ and setting $a_{1}=1, b_{1}=-1$, and $b_{2}=-q(r=1, s=2)$ in $[4$, Theorem 2.2], we find that

$$
-\frac{\left(q^{2} ; q^{2}\right)_{\infty}^{6}}{(q)_{\infty}^{2}\left(q^{4} ; q^{4}\right)_{\infty}^{4}} \sum_{n=-\infty}^{\infty} \frac{q^{n^{2}+3 n+1}}{\left(1+q^{2 n+1}\right)^{2}}+2 \sum_{\substack{n=-\infty \\ n \neq 0}}^{\infty} \frac{q^{n^{2}+2 n}}{\left(1+q^{2 n}\right)^{2}}=\frac{1}{2} \varphi(q) \varphi^{3}(-q)-\frac{1}{2} .
$$

Lastly, we also have that

$$
\begin{aligned}
2 \sum_{\substack{n=-\infty \\
n \neq 0}}^{\infty} \frac{q^{n^{2}+n}}{\left(1+q^{2 n}\right)^{2}} & =\sum_{\substack{n=-\infty \\
n \neq 0}}^{\infty} \frac{q^{n^{2}+n}}{1+q^{2 n}} \\
& =\sum_{\substack{n=-\infty \\
n \neq 0}}^{\infty} \frac{(-1)^{n} q^{n^{2}+n}}{1+q^{2 n}}+2 \sum_{n=-\infty}^{\infty} \frac{q^{(2 n+1)^{2}+(2 n+1)}}{1+q^{2(2 n+1)}} \\
& =\frac{1}{2} \varphi^{2}\left(-q^{2}\right)-\frac{1}{2}+4 \sum_{n \geqslant 0} \frac{q^{(2 n+1)^{2}+(2 n+1)}}{1+q^{2(2 n+1)}}
\end{aligned}
$$


where we have the last equality by using the following identity, which is the case $r=0$ and $s=1$ of [4, Theorem 2.1],

$$
\frac{(q)_{\infty}^{2}}{[a]_{\infty}}=\sum_{n=-\infty}^{\infty} \frac{(-1)^{n} q^{n(n+1) / 2}}{1-a q^{n}} .
$$

By (4.7), (4.8), and (4.9), we now need to prove that

$$
\varphi(q) \varphi^{3}(-q)+2 \varphi(-q) \varphi\left(q^{2}\right)+\varphi^{2}\left(-q^{2}\right)-4 \equiv 0 \quad(\bmod 8) .
$$

The identities (4.1), (4.2), and (4.3) will give us

$$
\begin{aligned}
\varphi(q) \varphi^{3}(-q) & =\varphi^{2}\left(-q^{2}\right)\left(\varphi^{2}\left(q^{2}\right)-4 q \psi^{2}\left(q^{4}\right)\right) \\
& =\left(\varphi\left(-q^{2}\right) \varphi\left(q^{2}\right)\right)^{2}-4 q\left(\varphi\left(-q^{2}\right) \psi\left(q^{4}\right)\right)^{2} \\
& =\varphi^{4}\left(-q^{4}\right)-4 q \psi^{4}\left(-q^{2}\right) \\
& \equiv 1-4 q \sum_{n \geqslant 0} \frac{\left(1-q^{8 n+4}\right) q^{4 n^{2}+4 n}}{\left(1-q^{4 n+2}\right)^{2}} \quad(\bmod 8) \\
& \equiv 1-4 \sum_{n \geqslant 0} q^{(2 n+1)^{2}} \quad(\bmod 8) .
\end{aligned}
$$

From the definition of $\varphi(q)$ and (4.1), we obtain that

$$
\begin{aligned}
& 2 \varphi(-q) \varphi\left(q^{2}\right)+\varphi^{2}\left(-q^{2}\right) \\
& =\varphi(-q)\left(2 \varphi\left(q^{2}\right)+\varphi(q)\right) \\
& =\left(1+2 \sum_{n \geqslant 1}(-1)^{n} q^{n^{2}}\right)\left(3+4 \sum_{n \geqslant 1} q^{2 n^{2}}+2 \sum_{n \geqslant 1} q^{n^{2}}\right) \\
& \equiv 3+4 \sum_{n \geqslant 1} q^{(2 n-1)^{2}}+4 \sum_{n \geqslant 1} q^{2 n^{2}}+4\left(\sum_{n \geqslant 1}(-1)^{n} q^{n^{2}}\right)\left(\sum_{n \geqslant 1} q^{n^{2}}\right) \quad(\bmod 8) \\
& =3+4 \sum_{n \geqslant 1} q^{(2 n-1)^{2}}+4 \sum_{n \geqslant 1}\left(1+(-1)^{n}\right) q^{2 n^{2}}+4 \sum_{n>m \geqslant 1}\left((-1)^{n}+(-1)^{m}\right) q^{n^{2}+m^{2}} \\
& \equiv 3+4 \sum_{n \geqslant 1} q^{(2 n-1)^{2}} \quad(\bmod 8) .
\end{aligned}
$$

The last two congruences (4.10) and (4.11) imply the desired result.

Also, we have the following congruences between $D$-rank and $M_{2}$-rank moments by Theorem 3 and (1.2).

Corollary 9. For all integers $n \geqslant 0$,

$$
\begin{array}{ll}
\bar{N}_{2}(3 n+i) & \equiv \overline{N 2}_{2}(3 n+i) \quad(\bmod 3), \quad \text { for } i=1,2, \\
\bar{N}_{2}(4 n+1) \equiv \overline{N 2}_{2}(4 n+1) & (\bmod 4) .
\end{array}
$$




\section{Acknowledgements}

The author would like to thank the referees for their careful reading and helpful comments.

\section{References}

[1] G. E. Andrews, The Ramanujan-Dyson Identities and George Beck's Congruence Conjectures, Int. J. Number Theory 17 (2021), 239-249.

[2] G. E. Andrews and F. G. Garvan, Dyson's crank of a partition, Bull. Amer. Math. Soc. (N.S.) 18 (1988), 167-171.

[3] A. O. L. Atkin and P. Swinnerton-Dyer, Some properties of partitions, Proc. London Math. Soc. (3) 4 (1954), 84-106.

[4] S. H. Chan, Generalized Lambert Series Identities, Proc. London Math. Soc. 91 (2005), 598-622.

[5] S. H. Chan, R. Mao, and R. Osburn, Variations of Andrews-Beck type congruences, J. Math. Anal. Appl. 495 (2021), Article 124771.

[6] S. Chern, Weighted partition rank and crank moments. I. Andrews-Beck type congruences, preprint.

[7] S. Chern, Weighted partition rank and crank moments. III. A list of Andrews-Beck type congruences modulo 5, 7, 11 and 13, Int. J. Number Theory 18 (2022), 141-163.

[8] F. J. Dyson, Some guesses in the theory of partitions, Eureka (Cambridge) 8 (1944), $10-15$.

[9] M. D. Hirschhorn and J. Sellers, Arithmetic properties for overpartitions, J. Comb. Math. Comb. Comput. 53 (2005), 65-73.

[10] C. Jennings-Shaffer, Higher order SPT functions for overpartitions, overpartitions with smallest part even, and partitions with smallest part even and without repeated odd parts, J. Number Theory 149 (2015), 285-312.

[11] J. Lovejoy, Rank and conjugation for the Frobenius representation of an overpartition, Ann. Comb. 9 (2005), 321-334.

[12] J. Lovejoy, Rank and conjugation for a second Frobenius representation of an overpartition, Ann. Comb. 12 (2008), 101-113. 